\title{
Knowledge Discovery Workflows in the Exploration of Complex Astronomical Datasets
}

\section{Raffaele D'Abrusco ${ }^{1}$, Giuseppina Fabbiano ${ }^{1}$, Omar Laurino ${ }^{1}$ and Francesco Massaro ${ }^{2}$}

${ }^{1}$ Harvard-Smithsonian Center for Astrophysics - Cambridge (MA), 02138 - Garden Street 60

${ }^{2}$ SLAC National Laboratory and Kavli Institute for Particle Astrophysics and Cosmology, 2575 Sand Hill Road, Menlo Park, CA 94025, USA

\begin{abstract}
The massive amount of data produced by the recent multi-wavelength large-area surveys has spurred the growth of unprecedentedly massive and complex astronomical datasets that are proving the traditional data analysis techniques more and more inadequate. Knowledge discovery techniques, while relatively new to astronomy, have been successfully applied in several other quantitative disciplines for the determination of patterns in extremely complex datasets. The concerted use of different unsupervised and supervised machine learning techniques, in particular, can be a powerful approach to answer specific questions involving high-dimensional datasets and degenerate observables. In this paper I will present CLaSPS, a data-driven methodology for the discovery of patterns in high-dimensional astronomical datasets based on the combination of clustering techniques and pattern recognition algorithms. I shall also describe the result of the application of CLaSPS to a sample of a peculiar class of AGNs, the blazars.
\end{abstract}

Keywords. surveys, data-mining, AGNs, blazars, WISE, $\gamma$-ray

\section{CLaSPS}

The Clustering-Labels-Score Pattern Spotter (CLaSPS) method (D'Abrusco et al. (2012)) for the discovery of patterns in complex astronomical feature spaces is based on unsupervised clustering techniques (Hastie et al. (2009)), complemented by additional data, the labels, employed to characterize the content of different clusters. The labels are used to characterize the content of the set of the clusters determined in the feature space. Previously, some of the authors ((D'Abrusco et al. (2009)) and (Laurino et al. (2011))) have used the same approach for the selection of optical candidate quasars from photometric datasets and the determination of photometric redshifts employing. CLaSPS has generalized this method extended to multiple labels, both numerical and categorial. The originality of CLaSPS lies in the criterion used to select the interesting aggregation of sources in the feature space that show correlations with the labels distribution. The quantitative diagnostics used to select such clusterings is called the score. For a generic clustering of the dataset in the feature space, the score of the $i$-th cluster is defined as:

$$
S_{i}=\sum_{j=1}^{M^{(L)}-1}\left\|f_{i j}-f_{i(j-1)}\right\|
$$

where $f_{i j}$ is the fraction of the $i$-th cluster members with values of the label falling in the $j$-th bin (or class) and the sum is over all labels bins. The total score $s$ of the clustering follows: 


$$
S_{\mathrm{tot}}=\frac{1}{N_{\mathrm{clust}}} \cdot \sum_{i=1}^{N_{\mathrm{clust}}} S_{i}=\frac{1}{N_{\mathrm{clust}}} \sum_{i=1}^{N_{\mathrm{clust}}}\left(\sum_{j=1}^{M^{(j)}-1}\left\|f_{i j}-f_{i(j+1)}\right\|\right)
$$

The values of the scores are then used to select the clustering(s) showing the largest degree of correlation between the label classes and clustering membership distributions (left plot in Fig. 1). The effectiveness of the score has been assessed on simulated clusterings before the application to real astronomical datasets.
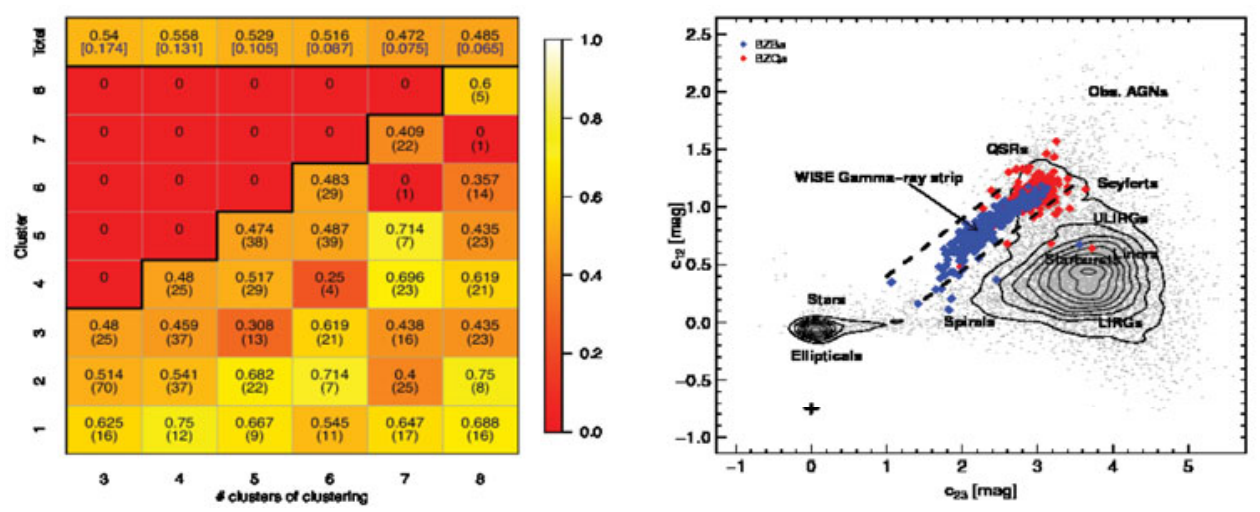

Figure 1. Left: map of the scores for the clusterings of the blazars experiment described in Sec. 2 as a function of the total number of clusters in each clustering. Right: projection of the blazars WISE locus, discovered by CLaSPS, onto the WISE [4.6]-[12] vs [3.4]-[4.6] color-color plane.

\section{Application to Blazars}

One of the first applications of CLaSPS, involving a sample of bona fide blazars in a feature space generated by broad-band color from mid-infrared to far ultraviolet, has led to the discovery of a previously unknown pattern followed by the blazars in the mid-infrared color space generated by the WISE magnitudes (D'Abrusco et al. (2012)). CLaSPS has been applied to the distribution of blazars in the 9-dimensional colors feature space using as labels, among other observables, the blazars spectral classification in BL Lacs and Flat Spectrum Radio Quasars, and the detection of $\gamma$-ray emission. CLaSPS determined a significant pattern for $\gamma$-ray emitting blazars of both spectral types in the three dimensional WISE colors space, revealing that this class of extragalactic sources occupy a peculiar and narrow locus in this feature space. The projection of the 3D WISE blazars locus color plane is shown in the right plot in Fig. 1. This discovery has also been used to devise a method for the selection of WISE candidate blazars that has been already applied to different sample of high-energy unidentified sources (the application to the unidentified $\gamma$-ray sources from Fermi in (Massaro et al. (2012)).

\section{References}

D'Abrusco, R., Longo, G., \& Walton, N. A. 2009, MNRAS, 396, 223

D'Abrusco, R., Massaro, F., Ajello, M., et al. 2012, ApJ, 748, 68

D'Abrusco, R., Fabbiano, G., Djorgovski, G., et al. 2012, ApJ, 755, 92

Hastie, T., Tibshirani, R., \& Friedman, J. 2009, The Elements of statistical learning, Springer.

Laurino, O., D'Abrusco, R., Longo, G., \& Riccio, G. 2011, MNRAS, 418, 2165

Massaro, F., D'Abrusco, R., Tosti, G., et al. 2012, ApJ, 750, 138 